\title{
Heat shock protein X purified from Mycobacterium tuberculosis enhances the efficacy of dendritic cells-based immunotherapy for the treatment of allergic asthma
}

\author{
Hye-young Kim ${ }^{1, \#}$, Hyun Kyu Kang ${ }^{2, \#}$, Joon Cho ${ }^{3, \#}$, In Duk Jung ${ }^{4}$, Gun Young Yoon ${ }^{4}$, Min-Goo Lee ${ }^{5}$, Sung Jae Shin ${ }^{6}$, Won \\ Sun Park, Jong-Hwan Park ${ }^{8}$, Seung-Wook Ryu ${ }^{9}$, Yeong-Min Park ${ }^{4, *}$ E Ji Chang You $^{10, *}$ \\ ${ }^{1}$ Department of Pediatrics, Pusan National University School of Medicine, Medical Research Institute of Pusan National University \\ Hospital, Pusan 602-739, ${ }^{2}$ Department of Microbiology and Immunology, School of Medicine, Pusan National University, ${ }^{3}$ Department of \\ Neurosurgery, Konkuk University Hospital, Seoul 380-701, ${ }^{4}$ Department of Immunology, Lab of Dendritic Cell Differentiation \& \\ Regulation, KU open innovation center and School of Medicine, Konkuk University, Chungju 380-701, ${ }^{5}$ Department of Physiology, College \\ of Medicine, Korea University, Seoul 136-705, ${ }^{6}$ Department of Microbiology, Institute for Immunology and Immunological Diseases, \\ Yonsei University College of Medicine, Seoul 120-752, ${ }^{7}$ Department of Physiology, School of Medicine, Kangwon National University, \\ Chuncheon 200-701, ${ }^{8}$ Department of Biochemistry, College of Medicine, Konyang University, Daejeon 302-711, ${ }^{9}$ Cell Signaling and \\ Bioimaging Laboratory, Department of Bio and Brain Engineering, KAIST, Daejeon 305-701, ${ }^{10}$ National Research Laboratory of Molecular \\ Virology, Department of Pathology, School of Medicine, The Catholic University of Korea, Seoul 137-701, Korea
}

\begin{abstract}
Dendritic cells play an important role in determining whether naïve $\mathrm{T}$ cells mature into either Th1 or Th2 cells. We determined whether heat-shock protein X ( $\mathrm{HspX})$ purified from Mycobacterium tuberculosis regulates the Th1/Th2 immune response in an ovalbumin (OVA)-induced murine model of asthma. HspX increased interferon-gamma, IL-17A, -12 and transforming growth factor (TGF) $\beta$ production and T-bet gene expression but reduced IL-13 production and GATA-3 gene expression. HspX also inhibited asthmatic reactions as demonstrated by an increase in the number of eosinophils in bronchoalveolar lavage fluid, inflammatory cell infiltration in lung tissues, airway luminal narrowing, and airway hyper-responsiveness. Furthermore, HspX enhanced OVA-induced decrease of regulatory $T$ cells in the mediastinal lymph nodes. This study provides evidence that HspX plays critical roles in the amelioration of asthmatic inflammation in mice. These findings provide new insights into the immunotherapeutic role of HspX with respect to its effects on a murine model of asthma. [BMB Reports 2015; 48(3): 178-183]
\end{abstract}

*Corresponding authors. Ji Chang You, Tel: +82-2-2258-7312; Fax: +82-2-2258-7790; E-mail: jiyou@catholic.ac.kr; Yeong-Min Park, Tel: +82-2-2049-6158; Fax: +82-2-2049-6192; E-mail: immun3023 @kku.ac.kr.

${ }^{\#}$ These authors contributed equally to this work.

http://dx.doi.org/10.5483/BMBRep.2015.48.3.257

Received 4 December 2014, Revised 19 December 2014, Accepted 26 December 2014

Keywords: Asthma, Dendritic cells, GATA-3, HspX, Mycobacterium tuberculosis, T-bet

\section{INTRODUCTION}

Asthma is the most common chronic airway inflammatory disease, and is characterized by persistent airway hyperresponsiveness (AHR) and airway inflammation resulting from cellular and molecular responses. It is thought to be caused by a combination of genetic and environmental insults, such as biologically active allergens, air pollutants, tobacco smoke, viruses, and stress $(1,2)$. As an allergic asthmatic model, ovalbumin (OVA)-induced asthma is characterized by increased AHR and airway inflammation, and is closely associated with the accumulation of inflammatory cells such as eosinophils, neutrophils, and lymphocytes in the airway and lung tissues (3). These cells infiltrate the airways and lungs, and produce various mediators capable of inducing inflammation and AHR (4, 5). In addition, recruitment of these inflammatory cells into sites of inflammation is a pivotal event in the development and extension of airway inflammation (6).

Heat shock protein HspX (Rv2031; molecular weight 16 $\mathrm{kDa}$ ) is required for mycobacterial persistence within macrophages, and is dominantly expressed during static growth or under oxygen deprivation (7). In a recent study, Mycobacterium bovis bacillus Camette-Guerin (BCG), killed by extended freeze-drying (EFD), reduced AHR (8). In addition, M. tuberculosis (Rv0577) induced dendritic cell (DC) maturation and drove a Th1 immune response (9). This supported the potential use of mycobacterial proteins for Th1 polarization via DCs.

Through interaction with antigen-presenting cells expressing an antigen/major histocompatibility complex (MHC) class II complex, naïve $\mathrm{T}$ cells are able to differentiate into different types of effector cells, which can be distinguished by their function including cytokine production $(10,11)$. During the immune response, two phenotypes of helper T (Th) cell, Th1 
and Th2, predominate and are distinguished by the function of the cytokines they produce (12). Regulatory T (Treg) cells are able to inhibit the development of allergic Th2 response-producing interleukin (IL)-10 and transforming growth factor (TGF)- $\beta$, and can induce tolerance against harmless antigens such as self-antigens $(13,14)$. Naturally occurring $\mathrm{CD}^{+}$ $\mathrm{CD} 25^{+}$Treg cells expressing the FoxP3 gene derived from the thymus, and Treg cells induced by cytokines from various peripheral cells, could contribute to suppressive and regulatory events (15). Th2 cells produce IL-4, IL-5, and IL-13 and mediate several regulatory and effector functions $(13,16)$. The recruitment of eosinophils and production of mucus and allergen-specific IgE are also induced by these cytokines (17). In addition, subpopulations of Th cells provide a mechanism for the development of different immune response qualities, depending on the stimuli in the host environment. In contrast, Th1 cytokines such as interferon-gamma (IFN- $\gamma$ ) and IL-12p70 inhibit the development of allergic lung inflammation by downregulating the Th2 response $(18,19)$. Thus, therapeutic interventions that inhibit Th2 cytokine production and simultaneously enhance Th1 cytokine production may be useful in managing allergic asthma.
Trans-acting T-cell-specific transcription factor (GATA-3) is a protein that belongs to the GATA family of transcription factors. GATA-3 binds to the T cell receptor-alpha (TCR- $\alpha$ ) gene enhancer and regulates luminal epithelial cell differentiation in the mammary gland $(20,21)$. Thus, it is an important regulator of T cell development. In particular, GATA-3 has been shown to promote the production of cytokines such as IL-4, IL-5, and IL-13 from Th2 cells (21). The T-box transcription factor (Tbx21) gene is a member of the T-box subfamily of genes that forms a common DNA-binding domain (22). T-box genes encodes for transcription factors essential for regulation of developmental processes. Tbx21 is involved in the human ortholog of the mouse Tbx21/T-bet gene (23). The study of the Mouse show that Tbx21 gene is a vital transcriptional regulation for Th1 cell polarization that controls the expression of the hallmark Th1 cytokine IFN- $\gamma$ (24). Therefore, T-bet has emerged as a key regulator for driving naïve $\mathrm{T}$ cell differentiation into Th1 cells and inducing a Th1 immune response (25). Additionally, T-bet, the expression of which is induced in Th1 but not in Th2 cells, is dependent on signal transduction, and acts as a powerful transactivator of the IFN- $\gamma$ gene in several kinds of cells, including Th1 cells $(24,25)$.
A

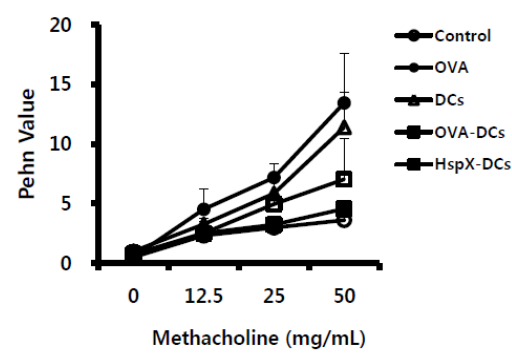

B

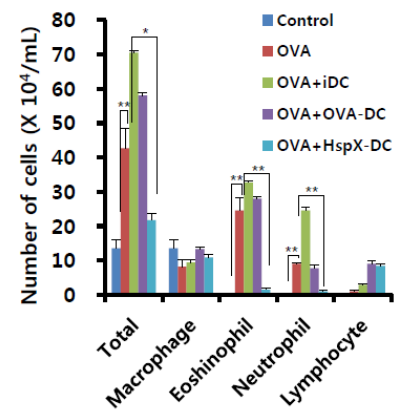

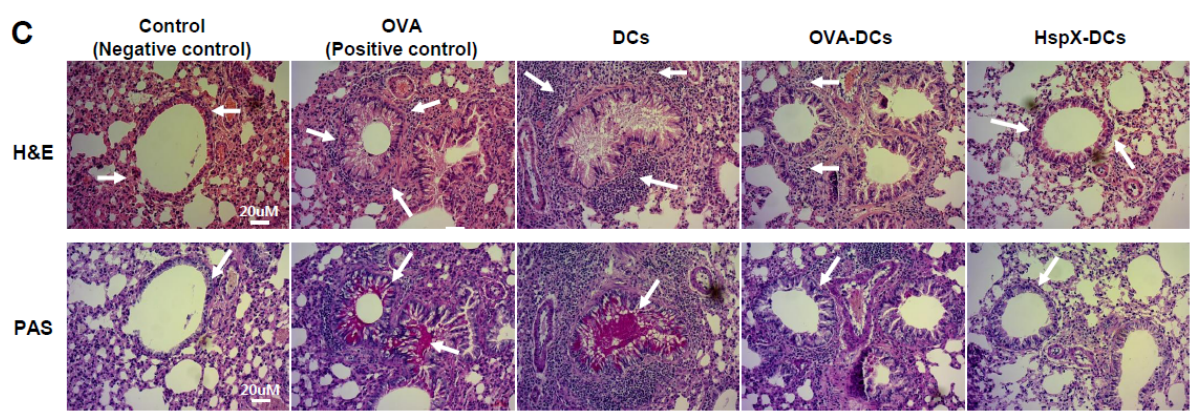

Fig. 1. Inhibition of airway responsiveness, lung inflammation, and inflammatory cell infiltration in mice treated by adoptive transfer of HspX-DCs. (A) Airway responsiveness was measured $24 \mathrm{~h}$ after the final challenge in saline-inhaled mice administered PBS (Control), OVA-sensitized mice administered saline (OVA), OVA-sensitized mice transferred with OVA-non pulsed DCs (DCs), OVA-sensitized mice transferred with OVA-pulsed DCs (OVA-DCs), and OVA-sensitized mice transferred with OVA-pulsed HspX-stimulated DCs (HspX-DCs). Enhanced pause (Penh) values were determined. Data are means \pm standard error of the mean (SEM) from three independent experiments. (B) Cells from bronchoalveolar lavage (BAL) fluid were collected and total differential inflammatory cell counts were conducted. (C) Lungs were removed $48 \mathrm{~h}$ after the final airway challenge. Sections were stained with haematoxylin and eosin $(\mathrm{H} \& \mathrm{E})$, and periodic acid-Schiff (PAS) $(400 \times)$. Representative results were of three mice per group from three independent experiments. Results are expressed as mean \pm SEM. 
Therefore, the effects of $M$. tuberculosis HspX on the expression levels of T-bet and GATA-3 in a murine allergic asthma model were determined.

In this study, we showed that the adoptive transfer of HspX-stimulated DCs (HspX-DCs) before airway OVA challenge resulted in significant inhibition of asthmatic reaction via increased Treg population, suggesting that $\mathrm{HspX}$ derived from M. tuberculosis could play a critical role in ameliorating asthma in mice.

\section{RESULTS}

Adoptive transfer of HspX-DCs inhibits development of AHR, lung inflammation, and inflammatory cell infiltration

The experimental protocol for the induction of the allergic asthmatic model is described in Supplementary Fig. 1. Following OVA sensitization, PBS, non-pulsed DCs with OVA (DCs), OVA-pulsed (OVA-DCs), or OVA-pulsed and HspX-stimulated DCs (HspX-DCs) were administered by i.t. 10 days prior to an inhaled OVA challenge. AHR was measured as the Penh value in response to increasing doses of methacholine $(0-50 \mathrm{mg} / \mathrm{ml})$. AHR was inhibited in mice that received HspX-DC (Fig. 1A). Also, the numbers of total cells, eosinophils, lymphocytes, and macrophages in BAL fluid increased $48 \mathrm{~h}$ after the last OVA challenge compared with the number of cells after saline inhalation (Fig. 1B). Histological analyses showed the typical pathological features of asthma in OVA-sensitized and OVAchallenged mice compared with control mice. The OVA-exposed mice displayed many inflammatory cells around the bronchioles, and mucin was also increased (Fig. 1C, upper panel). Mice treated with HspX-DC showed a marked decrease in inflammatory cell infiltration in the peribronchiolar and perivascular regions, and also showed a reduction of mucin (Fig. 1C, lower panel). Therefore, the increase in total lung inflammation and infiltration of inflammatory cells was significantly inhibited by the adoptive transfer of HspX-DCs.
These results indicate that the adoptive transfer of HspX-DCs inhibits OVA-induced AHR and antigen-induced inflammation in the lungs, and suppresses mucin production.

\section{HspX increases production of TNF- $\alpha$, IL-6, IL-12p70, IFN- $\gamma$, and IL-17 in BAL fluids of OVA-sensitized and OVA-challenged mice}

BAL fluids of control mice and OVA-sensitized and OVA-challenged mice were obtained $48 \mathrm{~h}$ after final airway challenge with OVA. In the BAL fluids of mice subjected to airway challenge with OVA, the levels of the Th2 cytokine IL-13 were slightly higher than those in the control mice (Fig. 2D). Also, inflammatory cytokine TNF- $\alpha$ levels were increased compared with those in the control mice (Fig. 2A). In contrast, the levels of the Th1 cytokines IFN- $\gamma$ (Fig. 2B) and IL-12p70 (Fig. 2C), the Th17 cytokine IL-17A (Fig. 2E), and the pro-inflammatory cytokine IL-6 (Fig. 2F) were decreased in comparison with those of the control mice. However, the adoptive transfer of HspX-DCs reversed these phenomena (Fig. 2). As shown in Fig. 3, the levels of IL-13 and TNF- $\alpha$ in mice subjected to adoptive transfer with HspX-DCs were decreased compared with OVA-sensitized and OVA-challenged mice that had not received HspX-DCs. Furthermore, the levels of the Th1 cytokines IFN- $\gamma$ and IL-12p70, the Th17 cytokine IL-17A, and the pro-inflammatory cytokine IL-6 were significantly higher in mice adoptively transferred with HspX-DCs than in OVA-sensitized and OVA-challenged mice that were not transferred with HspX-DCs or did not receive OVA-DCs. These results indicate that HspX-DCs from M. tuberculosis might have an immune modulatory function that drives $T$ cell polarization by interaction with HspX-stimulated dendritic cells, leading to the amelioration of asthma.

\section{Adoptive transfer of HspX-DCs decreases IgE levels in the serum of allergic asthma model mice}

Since Th2 cytokines promote airway inflammation in asthma
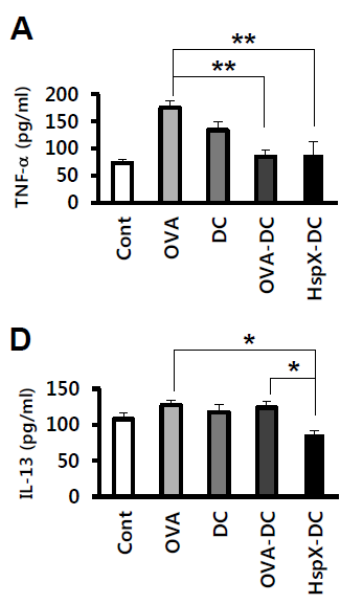

B

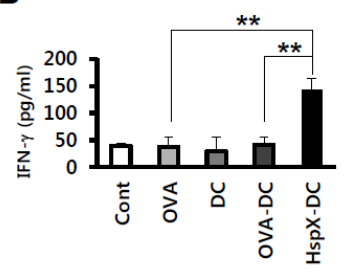

E

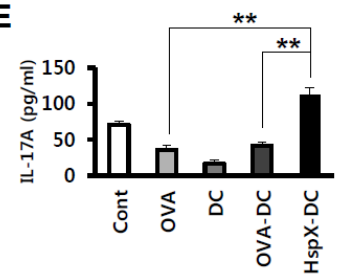

C

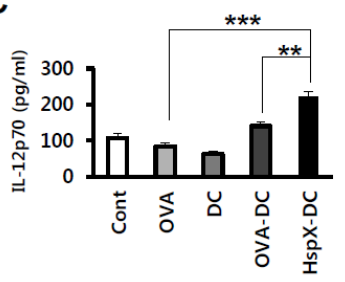

$\mathbf{F}$

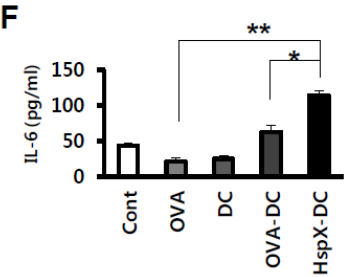

Fig. 2. Adoptive transfer of HspX-DCs modulates cytokines. Mice were treated as described in the Materials and Methods section. BAL fluid from each group of mice was obtained $48 \mathrm{~h}$ after the final airway challenge. TNF- $\alpha$ (A), IFN- $\gamma$ (B), IL-12p70 (C), IL-13 (D), IL-17A (E), and IL-6 (F) levels in the BAL fluid were measured by enzymelinked immunosorbent assay (ELISA) according to the manufacturer's instructions. Results are means \pm SEM from three experiments. Statistical significance was assessed by Student's paired t test, which was used to determine differences between two groups. The Turkey-Kramer test was used for comparisons between multiple groups. ${ }^{*} \mathrm{P}<0.05,{ }^{* *} \mathrm{P}<$ 0.01 , and $* * * P<0.001$. 
A
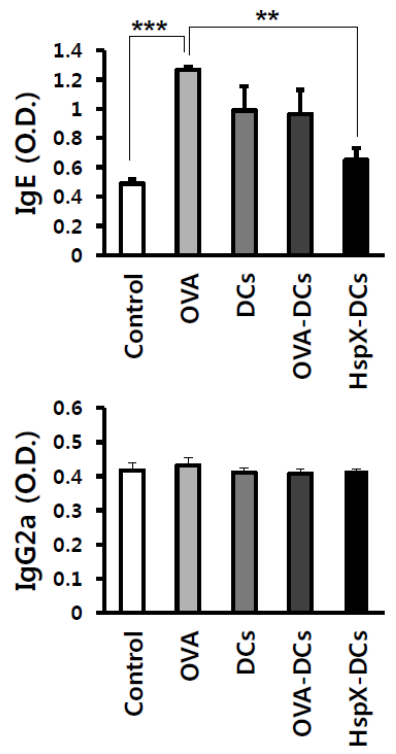

B
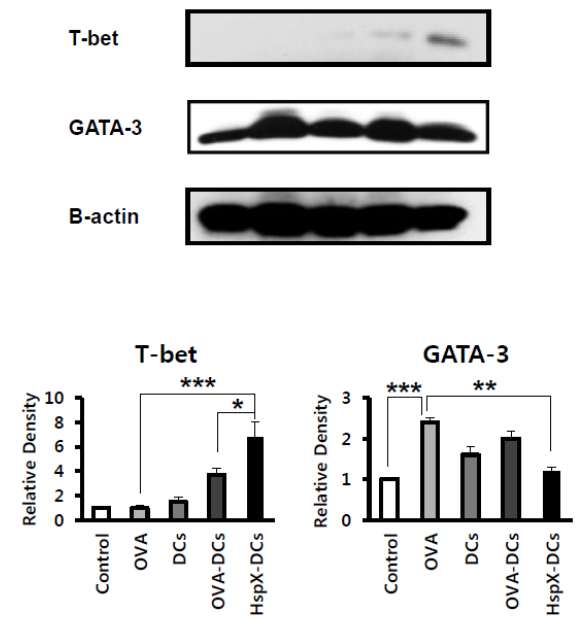

Fig. 3. Effect of adoptive transfer of HspX-DCs on the expression level of GATA-3 and T-bet, and immunoglobulin production. (A) Blood was collected by cardiac puncture, and serum IgE and IgG2a levels were analyzed using ELISA $(\mathrm{n}=5)$. Data are means \pm SEM from three experiments. ${ }^{* * P}<$ 0.01 and $* * * P<0.001$. (B) Samples were obtained 2 days after the last OVA challenge from mice that received PBS (control), non-pulsed DCs with OVA (DCs), OVA pulsed DCs (OVA-DCs), or OVA pulsed and HspX -stimulated DCs (HspX-DCs), and were subjected to western blot analysis with $\alpha$-GATA-3 or $\alpha$-T-bet antibody.

A

CD4+ $\mathrm{T}$ cells in $\mathrm{mLN}$

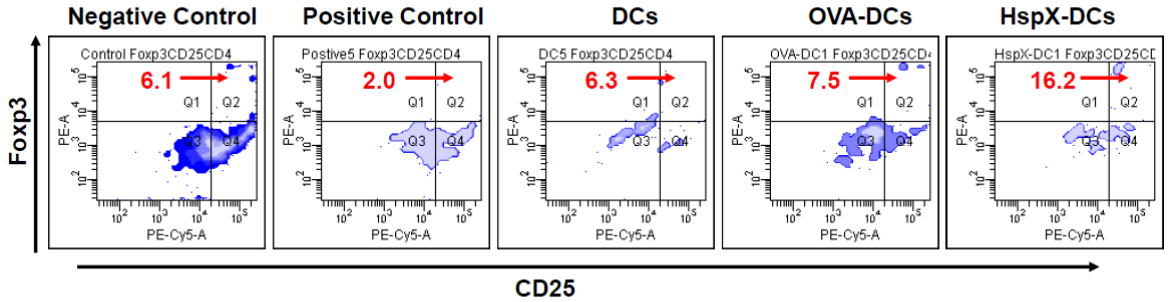

B

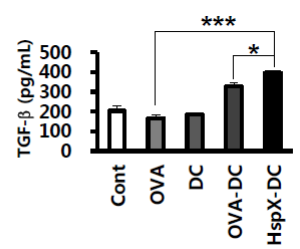

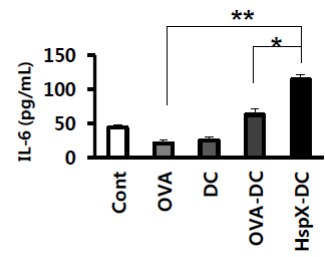

Fig. 4. Effect of HspX-DCs adoptive transfer on Treg cells and production of TGF- $\beta$ in allergic asthma mouse model. (A) Mediastinal lymph nodes were obtained 2 days after the last OVA challenge, and treated with collagenase IV. Cells were stained with CD4, CD25 and Foxp3 antibodies. Representative results of three independent experiments. (B) BAL fluid from each group of mice was obtained $48 \mathrm{~h}$ after the final airway challenge. TGF- $\beta$ levels in the BAL fluid were measured by ELISA according to the manufacturer's instructions. Results are expressed as mean \pm SEM. $* P<0.05, * * P<0.01$ and $* * * P<0.001$. by increasing IgE levels, which in turn favors the Th2 inflammatory pathway, the extent to which the adoptive transfer of HspX-DCs modulates serum IgE levels in OVA-induced allergic asthmatic mice was determined. As shown in Fig. 4, serum IgE levels in OVA-sensitized and OVA-challenged mice were significantly higher than those in the control mice. Mice that received HspX-DCs by adoptive transfer had significantly decreased serum IgE levels, but serum IgG2a levels were unaffected (Fig. 3A). These data indicate that the adoptive transfer of HspX-DCs into mice modulates the balance of Th1 and Th2 towards Th1 in an OVA-induced allergic asthma model. The observed increases in the number of inflammatory cells were significantly inhibited by the adoptive transfer of HspX-DCs.

\section{HspX-DCs reduce GATA-3 expression in lung tissue of OVA-sensitized and OVA-challenged mice}

Western blot analysis showed that GATA-3 expression was significantly increased in lung tissues $48 \mathrm{~h}$ after OVA-challenge compared with control tissues, but the level of T-bet expression was not. However, the adoptive transfer of HspX-DCs significantly inhibited the increase in GATA-3 expression (Fig. 3B). In contrast, the adoptive transfer of HspX-DCs induced an 
increase in T-bet expression (Fig. 3B). These data indicate that the adoptive transfer of HspX-DCs into allergic asthma mice modulates gene expression levels, such as the increase of the Th1 gene T-bet and the decrease of the Th2 gene GATA-3, and ameliorates allergic asthma in mice.

\section{Adoptive transfer of HspX-DCs increases regulatory T cells in mediastinal lymph nodes and TGF- $\beta$ production in allergic asthma mouse model}

Because adoptive transfer of HspX-DCs into allergic asthma mice modulates the Th1/Th2 immune balance and decreases the allergic immune response, we next investigated the effect of the adoptive transfer of HspX-DCs on regulatory $\mathrm{T}$ cells in mediastinal lymph nodes. As shown in Fig. 4A, OVA-sensitization and OVA-challenge (Positive Control group) decreased the Treg cell population. However, the adoptive transfer of HspX-DCs increased the number of Treg cells compared with the positive control mice. Therefore, we investigated TGF- $\beta$ and IL-6 production in BAL fluids, which is important to regulatory $\mathrm{T}$ cells function and maintenance. OVA-sensitization and OVA-challenge decreased TGF- $\beta$ and IL- 6 production in the positive control mice with asthma, but the adoptive transfer of HspX-DCs increased its production (Fig. 4B). Taken together, these data suggest that the adoptive transfer of HspX-DCs can influence the Treg cell population and the Th1/Th2 immune balance, and can ameliorate the allergic asthma response.

\section{DISCUSSION}

This study demonstrates that immunotherapy with HspX-DCs by adoptive transfer can modulate airway inflammation in a murine model of asthma. Remarkably, the adoptive transfer of HspX-DCs profoundly inhibited allergic asthmatic reactions, including recruitment of inflammatory cells into the airway and lung inflammation. It was also found that HspX-DC adoptive transfer regulates the balance of Th1 and Th2 immune responses by modulating the levels of T-bet and GATA-3, as well as the production of the cytokine IL-17A.

The role of HspX is involved in slowing the growth of $M$. tuberculosis in vivo immediately after infection, showing the HspX plays a pivotal role an antigenic target of the immune response during latent tubercle bacillus (TB) infection. T cells from TB patients recognizing the $\mathrm{HspX}$ protein indicated that Th0 differentiate into Th1 after chemotherapy, indicating their potential to induce protective T-cell responses (7).

Allergic asthma induced by allergens such as OVA is recognized as a disease caused by chronic airway inflammation, which is characteristically associated with the infiltration of lymphocytes, eosinophils, and neutrophils into the bronchial wall. In this study, it was observed that OVA-induced allergic asthma increased the infiltration of eosinophils. However, the adoptive transfer of HspX-DCs significantly inhibited the asthmatic reaction. T-bet, a member of the T-box family of tran- scription factors, is a master determinant of the Th1 cell lineage. T-bet-deficient mice lack the Th1 immune response (26), which inhibits allergic responses (27). In addition, the ectopic expression of T-bet in murine Th2 cells directs the activation of IFN- $\gamma$ and the upregulation of IL-12R $\beta$ (28). GATA-3 belongs to the GATA family of transcription factors. This family has six members (GATA-1 to GATA-6), which were identified in birds and have homologues in mammals. Based on their expression profiles and structures, GATA proteins may be classified as either hematopoietic (GATA-1 to GATA-3) or non-hematopoietic (GATA-4 to GATA-6) (29). The GATA-3 expression level in naïve $\mathrm{CD} 4^{+} \mathrm{T}$ cells is low. However, the expression of GATA-3 depends on T cell lineage; it is markedly upregulated in cells differentiating along the Th2 pathway, while it is downregulated in cells differentiating along the Th1 pathway (21). These data demonstrate that the adoptive transfer of HspX-DCs into mice inhibits the increase in GATA-3 expression in OVA-sensitized and OVA-challenged mice (Fig. 3). Therefore, it could be concluded that adoptive transfer of HspX-DCs is a selective tool to simultaneously suppress GATA-3 and increase T-bet expression in asthmatic reactions in vivo.

Furthermore, the production of Th1 and Th2 cytokines was examined in $\mathrm{CD}^{+}{ }^{+} \mathrm{T}$ cells. The adoptive transfer of HspX-DCs inhibited the increase in the level of IL-13, a Th2 cytokine produced in OVA-sensitized and OVA-challenged mice. On the other hand, HspX-DC transfer increased the level of IL-12p70 and IFN- $\gamma$, a Th1 cytokine produced in mice that have adoptively received HspX-DCs. Taken together, these results strongly indicate that HspX-DCs reduce allergic airway inflammation and hyperresponsiveness by modulating the balance of Th1 and Th2 immune responses through the suppression of GATA-3 expression and induction of T-bet expression.

Treg cells can inhibit the development of the allergic Th2 response producing IL-10 and TGF- $\beta$, and induce tolerance against harmless antigens such as self-antigens. Thus, Treg cells may contribute to suppressive and regulatory events in the immune response. In our experiment, OVA-sensitization and OVA-challenge decreased the Treg cell population. However, the adoptive transfer of HspX-DCs increased the number of Treg cells compared with the positive control mice. Also, TGF- $\beta$ production was increased by the adoptive transfer of HspX-DCs compared with the positive asthma control mice. Therefore, these data suggest that the adoptive transfer of HspX-DCs influences Treg cells and the Th1/Th2 balance, although the order in which these mechanisms are activated, and their effects on each other, remain unclear. In conclusion, the findings of this study suggest that immunotherapy using HspX-stimulated DC transfer might offer a new therapeutic approach to the treatment of allergic airway diseases.

\section{MATERIALS AND METHODS}

Detailed descriptions of all the methods used in this study are provided in the supplementary section. 


\section{ACKNOWLEDGEMENTS}

This study was supported by the National Research Foundation of Korea (NRF) grants funded by the Korea government (NRF2012R1A2A1A03008433 and NRF-2013R1A2A2A01068353).

\section{REFERENCES}

1. Franchini $M$, Gilli $U$, Akens $M K$, Fellenberg RV and Bracher V (1998) The role of neutrophil chemotactic cytokines in the pathogenesis of equine chronic obstructive pulmonary disease (COPD). Vet Immunol Immunopathol 66, 53-65

2. Yawn BP (2008) Factors accounting for asthma variability: achieving optimal symptom control for individual patients. Prim Care Respir J 17, 138-147

3. Spicuzza L, Bonfiglio C and Polosa R (2003) Research applications and implications of adenosine in diseased airways. Trends Pharmacol Sci 24, 409-413

4. Mapp CE, Boschetto P, Zocca E et al (1987) Pathogenesis of late asthmatic reactions induced by exposure to isocyanates. Bull Eur Physiopathol Respir 23, 583-586

5. Bousquet J, Chanez P, Lacoste JY et al. (1990) Eosinophilic inflammation in asthma. N Engl J Med 323, 1033-1039

6. Kay AB (1991) Asthma and inflammation. J Allergy Clin Immunol 87, 893-910

7. Geluk A, Lin MY, van Meijgaarden KE et al (2007) T-cell recognition of the HspX protein of Mycobacterium tuberculosis correlates with latent $M$. tuberculosis infection but not with $M$. bovis BCG vaccination. Infect Immun 75, 2914-2921

8. Lagranderie M, Abolhassani M, Vanoirbeek J et al (2008) Mycobacterium bovis BCG killed by extended freeze-drying reduces airway hyperresponsiveness in 2 animal models. J Allergy Clin Immunol 121, 471-478

9. Byun EH, Kim WS, Kim JS et al (2012) Mycobacterium tuberculosis Rv0577, a novel TLR2 agonist, induces maturation of dendritic cells and drives Th1 immune response. FASEB J 26, 2695-2711

10. Furuta K, Ishido S and Roche PA (2012) Encounter with antigen-specific primed CD4 T cells promotes MHC class II degradation in dendritic cells. Proc Natl Acad Sci U S A 109, 19380-19385

11. Wu Z, Rothwell L, Young JR, Kaufman J, Butter C and Kaiser P (2010) Generation and characterization of chicken bone marrow-derived dendritic cells. Immunology $129,133-145$

12. Zhang X, Brunner T, Carter L et al (1997) Unequal death in T helper cell (Th) 1 and Th2 effectors: Th1, but not Th2, effectors undergo rapid Fas/FasL-mediated apoptosis. J Exp Med 185, 1837-1849

13. Ozdemir C, Akdis M and Akdis CA (2009) T regulatory cells and their counterparts: masters of immune regulation. Clin Exp Allergy 39, 626-639

14. Hamza E, Akdis CA, Wagner B, Steinbach F and Marti E (2013) In vitro induction of functional allergen-specific CD4 + CD25high Treg cells in horses affected with insect bite hypersensitivity. Clin Exp Allergy 43, 889-901

15. Wing JB and Sakaguchi S (2014) Foxp3(+) T(reg) cells in humoral immunity. Int Immunol 26, 61-69

16. Tanaka H, Komai M, Nagao K et al (2004) Role of interleukin-5 and eosinophils in allergen-induced airway remodeling in mice. Am J Respir Cell Mol Biol 31, 62-68

17. Corrigan CJ and Kay AB (1992) T cells and eosinophils in the pathogenesis of asthma. Immunol Today 13, 501-507

18. Iwamoto I, Nakajima H, Endo H and Yoshida S (1993) Interferon gamma regulates antigen-induced eosinophil recruitment into the mouse airways by inhibiting the infiltration of CD4+ T cells. J Exp Med 177, 573-576

19. Sur S, Lam J, Bouchard P, Sigounas A, Holbert D and Metzger WJ (1996) Immunomodulatory effects of IL-12 on allergic lung inflammation depend on timing of doses. J Immunol 157, 4173-4180

20. Ho IC, Vorhees P, Marin N et al (1991) Human GATA-3: a lineage-restricted transcription factor that regulates the expression of the T cell receptor alpha gene. EMBO J 10, 1187-1192

21. Zhou M and Ouyang W (2003) The function role of GATA-3 in Th1 and Th2 differentiation. Immunol Res 28, 25-37

22. Szabo SJ, Kim ST, Costa GL, Zhang X, Fathman CG and Glimcher LH (2000) A novel transcription factor, T-bet, directs Th1 lineage commitment. Cell 100, 655-669

23. Miller SA and Weinmann AS (2010) Molecular mechanisms by which T-bet regulates T-helper cell commitment. Immunol Rev 238, 233-246

24. Szabo SJ, Sullivan BM, Stemmann C, Satoskar AR, Sleckman BP and Glimcher LH (2002) Distinct effects of T-bet in $\mathrm{TH} 1$ lineage commitment and IFN-gamma production in CD4 and CD8 T cells. Science 295, 338-342

25. Zheng J, Liu Y, Qin G et al (2011) Generation of human Th1-like regulatory CD4 + T cells by an intrinsic IFN-gamma- and T-bet-dependent pathway. Eur J Immunol 41, 128-139

26. Mullen AC, High FA, Hutchins AS et al (2001) Role of T-bet in commitment of TH1 cells before IL-12-dependent selection. Science 292, 1907-1910

27. Bian T, Yin KS, Jin SX et al (2006) Treatment of allergic airway inflammation and hyperresponsiveness by imiquimod modulating transcription factors T-bet and GATA-3. Chin Med J (Engl) 119, 640-648

28. Afkarian M, Sedy JR, Yang J et al (2002) T-bet is a STAT1-induced regulator of IL-12R expression in naive CD4 + T cells. Nat Immunol 3, 549-557

29. Gregoire JM and Romeo PH (1999) T-cell expression of the human GATA-3 gene is regulated by a non-lineage-specific silencer. J Biol Chem 274, 6567-6578

30. Jung ID, Jeong SK, Lee CM et al (2011) Enhanced efficacy of therapeutic cancer vaccines produced by co-treatment with Mycobacterium tuberculosis heparin-binding hemagglutinin, a novel TLR4 agonist. Cancer Res 71, 2858-2870

31. Jung ID, Kim HY, Park JW et al (2012) RG-II from Panax ginseng C.A. Meyer suppresses asthmatic reaction. BMB Rep 45, 79-84 